\title{
Pregnancy Outcome of Twin Pregnancy at Gandaki Medical College Teaching Hospital, Pokhara, Nepal
}

\author{
Tripathi $\mathbf{M}^{1^{*}}$, Shrestha $\mathbf{R}^{2}$ \\ ${ }^{1}$ Professor \& HOD, ${ }^{2}$ Assistant Professor, Department of Obstetrics \& Gynecology, \\ Gandaki Medical College \& Teaching Hospital, Pokhara, Nepal
}

\author{
Keywords \\ Complication, Pregnancy outcome, \\ Preterm delivery, Twin pregnancy.

\section{Corresponding author} \\ ${ }^{*}$ Dr. Malati Tripathi, \\ Professor \& Head \\ Department of Obstetrics \& Gynecology \\ Gandaki Medical College \& Teaching \\ Hospital, Pokhara, Nepal \\ E-mail:drmalatitripathi@hotmail.com
}

\begin{abstract}
Objectives: To evaluate maternal and neonatal complications and pregnancy outcomes of twin pregnancies.

Methods: The cross sectional study was conducted using retrospective data on the twin pregnancies with more than 28 weeks of gestational age. The study used data over a period of five years, from March 10, 2010 to March 9, 2015 in the Department of Obstetrics and Gynecology, GMC Teaching Hospital Pokhara.
\end{abstract}

Results: Of the 50 twin pregnancies, the most common maternal complication was preterm delivery (40\%). Other maternal complications were anemia (36\%), pregnancy induced hypertension (14\%), premature rupture of membranes $(14 \%)$, postpartum hemorrhage $(12 \%)$ and antepartum hemorrhage (6\%). Median gestational age at delivery was 37 weeks. Most common route of delivery was cesarean section (66\%). Most common neonatal complication was low birth weight (48\%) births first twin and second twin 56\%.

Conclusion: Twin pregnancy has high maternal and neonatal complications, especially preterm delivery that increases the risk of significant neonatal morbidity and mortality.

\section{INTRODUCTION}

Twin pregnancy is usually associated with greater maternal and fetal risks than singleton pregnancy. Twin pregnancy still warrants special attention from obstetricians. Throughout the world, the prevalence of twin births varies considerably between two to 20 per 1000 births $^{1}$.

The dramatic rise in multiple gestations has been attributed to the increase use of ovulation inducing agents, use of assisted reproductive technologies, and a shift toward bearing children at older maternal ages when multiple gestations are more likely occur naturally². Multiple pregnancies are recognized as high risk pregnancy, associated with increased incidence of adverse pregnancy outcomes and risk for both maternal and fetal morbidity and mortality ${ }^{3}$.

Women with twin pregnancy have about two-fold increase in the risk of death compared with women with a singleton gestation. Babies born from twin birth pregnancies are more likely to result in premature birth than those from single pregnancies. About half of twins are born with a birth weight of less than 2,500 g $(5.5 \mathrm{lb})^{4}$. However, the chances of survival for very small twin babies are higher than for very small single babies ${ }^{5}$.

Major congenital abnormalities are more common in twin pregnancies as compared with singleton pregnancy. Twin to twin transfusion syndrome is a rare but potentially serious complication in identical twins these days. Survival rates are much higher due to early detection and also because of laser treatment, but the laser treatment is performed in few hospitals only, specialized in this procedure ${ }^{6}$.

Pre-eclampsia is two to three times more common in multiple than singleton pregnancy and it is likely to be more severe ${ }^{7}$. 


\section{METHODS}

This was a cross sectional study conducted on the twin pregnancies with more than 28 weeks of gestational age delivered at Gandaki Medical College Teaching Hospital, Pokhara, Nepal. This study used the retrospective data of 50 twin pregnancy cases in the period from March 10, 2010 to March 9, 2015.

The data retrieved from the medical records included demographic data, complications of pregnancy, and maternal and neonatal outcomes. A record form was used to collect data concerning maternal and neonatal parameters including maternal complications, antepartum complications, intrapartum presentations, neonatal outcomes, neonatal complications, and perinatal mortality.

\section{RESULTS}

A total of 50 twin pregnancies were delivered during the study period.

Table 1: Twin pregnancy in relation to maternal characteristics ( $n=50)$

\begin{tabular}{lcc}
\hline \multicolumn{1}{c}{ Maternal characteristics } & Frequency & Percentage \\
Maternal age in years & & \\
$18-20$ & 16 & $14 \%$ \\
$21-30$ & 27 & $32 \%$ \\
$31-40$ & & $54 \%$ \\
Parity & 8 & \\
Primi gravida & 17 & $34 \%$ \\
Multi gravida & 25 & $50 \%$ \\
Grand multigravida & \\
\hline
\end{tabular}

Higher percentage of women was in age group 31 - 40. Half of the women were of grand multi gravida.

Table 2: Mode of delivery in twin pregnancy

\begin{tabular}{lcc}
\hline \multicolumn{1}{c}{ Mode of delivery } & $\mathbf{1}^{\text {st }}$ twin $(\mathbf{n = 5 0 )}$ & $\begin{array}{c}2^{\text {nd }} \text { twin } \\
(\mathbf{n}=\mathbf{5 0})\end{array}$ \\
Spontaneous vaginal delivery & $20(40 \%)$ & $13(26 \%)$ \\
Assisted breech delivery & $1(2 \%)$ & $4(8 \%)$ \\
Instrumental vaginal delivery & $1(2 \%)$ & 0 \\
Cesarean section & $28(56 \%)$ & $28(56 \%)+5$ \\
\hline
\end{tabular}

The higher percentage of twins was delivered by cesarean section. Five cases of second twin were delivered by cesarian section after vaginal delivery of first twin. The indications were large size of second twin in two cases and prompt closer of the cervix after delivery of the first twin in three cases.
Table 3: Lie and presentation in twin pregnancy $(n=50)$

\begin{tabular}{lcc}
\hline \multicolumn{1}{c}{ Lie and presentation } & Frequency & Percentage \\
Both vertex & 28 & $56 \%$ \\
First vertex and $2^{\text {nd }}$ breech & 10 & $20 \%$ \\
First breech and $2^{\text {nd }}$ vertex & 7 & $14 \%$ \\
Both breech & 5 & $10 \%$ \\
First vertex and $2^{\text {nd }}$ transverse & 0 & $0 \%$ \\
Both transverse & 0 & $0 \%$ \\
\hline
\end{tabular}

$\mathrm{p}=0.629$ of $1^{\text {st }}$ twin and $2^{\text {nd }}$ twin was not significantly different. Fifty six percent of twins presented as both vertex presentation.

Table 4: Maternal complication and pregnancy outcome $(\mathrm{n}=50)$

\begin{tabular}{lcc}
\hline \multicolumn{1}{c}{ Maternal complication } & Frequency & Percentage \\
Pre term delivery & 20 & $40 \%$ \\
Anemia & 18 & $36 \%$ \\
Polyhydramnios & 8 & $16 \%$ \\
Pregnancy induced hypertension & 7 & $14 \%$ \\
Premature rupture of membrane & 7 & $14 \%$ \\
Postpartum hemorrhage & 6 & $12 \%$ \\
Antepartum hemorrhage & 3 & $6 \%$ \\
Placenta previa & 2 & $4 \%$ \\
Abruptio placenta & 1 & $2 \%$ \\
Cord prolapse & 1 & $2 \%$ \\
\hline
\end{tabular}

Pre term delivery was observed as most common maternal complication followed by anemia.

Table 5: Neonatal outcome

\begin{tabular}{lcc}
\hline \multicolumn{1}{c}{ Neonatal outcome } & $\mathbf{1}^{\text {st }}$ twin $(\mathbf{n = 5 0 )}$ & $2^{\text {nd }}$ twin $(\mathbf{n}=\mathbf{5 0 )}$ \\
Low birth weight & $22(44 \%)$ & $25(50 \%)$ \\
Very low birth weight & $2(4 \%)$ & $3(6 \%)$ \\
Apgar $<7$ in 5 minute & 0 & $1(2 \%)$ \\
Admission in NICU & $24(48 \%)$ & $31(62 \%)$ \\
Perinatal death & $2(4 \%)$ & $5(10 \%)$ \\
\hline
\end{tabular}

Low birth weight was more common outcome. Nearly half of the first twins were admitted in NICU and more than half of second twins were admitted in NICU. There was no twin-twin transfusion observed.

\section{DISCUSSION}

Twin pregnancy is high risk pregnancy associated with increased maternal morbidity, and increased perinatal morbidity and mortality. The highest incidence was found in women of age group $31-40$ years (54\%). The similar observation was found in the study conducted by Malik MS et $a l^{8}$.

In this study, $40 \%$ of the women presented with preterm Labor. Premature rupture of membranes occurred more frequently in twin pregnancy. Preterm labor and birth were frequent sequelae. Over distension of the uterus, polyhydramnios and premature rupture of the membranes 
were responsible for preterm labor.

Anemia was more frequent in twin pregnancy than in singleton pregnancy. Thirty six percent of women suffered with anemia in present study. The greater increase in blood volume compared with red cell mass decreased in hemoglobin concentration, producing a more pronounced decrease in hemoglobin compared with singleton pregnancy. Fetal demands in a twin pregnancy are greater, particularly for folate. Deficiency of folic acid leads to increased incidence of megaloblastic anaemia. During the antenatal period; anemia, preterm labor, PIH and abruption placentae were the major complicating factors. However, in a study done by Shahela Khatiq, anemia was the most common complication followed by preterm labor, $\mathrm{PIH}$ and intrauterine growth restriction in that order; in twin pregnancy ${ }^{9}$.

Hydramnios is more common in monozygotic twins and usually involves the second sac, and may cause significant abdominal discomfort for the mother. The incidence of pregnancy induced hypertension, pre-eclampsia, and eclampsia is increased in twin pregnancy. Antepartum hemorrhage as a result of placenta praevia and placental abruption is increased in twin pregnancy. The increase incidence of placenta praevia is due to bigger size of the placenta encroaching on the lower segment. The separation of normally situated placenta may be due to increased incidence of pre-eclampsia, sudden escape of liquor following rupture of membranes, folic acid deficiency, following delivery of first baby. APH was found in six percent of cases in the present study. In our study spontaneous vaginal delivery was more common for first twin (40\%), while for second twin it was $26 \%$. Cesarean section rate was $56 \%$ for first twin and $66 \%$ for second twin. In mode of delivery, cesarean section was the most common route of delivery.

Multiple pregnancy puts mother at risk of miscarriage, preeclampsia, APH, PPH, iron and folic acid deficiency anemia, polyhydramnios, preterm labor, PROM and increased rate of cesarean section ${ }^{10}$. Today many pregnancies are the result of subfertility therapy. By limiting the number of embryo transferred can reduce the risk of having multiples and so reduce the risk associated with multiple pregnancies $^{11}$.

The reduction of perinatal mortality of twins may be due to better antenatal care, early diagnosis, early detection of complications, tocolysis and steroid administration for management of preterm labor and better neonatal care.
According to gestational age at delivery, perinatal death was decreased when gestational age increased.

\section{CONCLUSIONS}

Twin pregnancy has high maternal complications such as preterm delivery and anemia of mothers; and neonatal complications like low birth weight, increased NICU admission and perinatal deaths.

\section{REFERENCES}

1. Doris MC. Multiple pregnancy. Baillieres Clin Obstet Gynaecol. 1990; 4: 109- 27.

2. Jewell SE, Yip R. Increasing trends in plural births in the United States. Obstet Gynecol. 1995; 85: 229-32.

3. Kauppila A, Jouppila P, Koivisto M, Moilanen I, Ylikorkala O. Twin pregnancy: A clinical study of 335 cases. Acta Obstet Gynecol Scand. 1975; 54: 5-11.

4. HFEA. Multiple pregnancies and birth: Considering the risks. London: Human Fertilisation and Embyrology Authority. 2006.

5. Cnattingius S, Haglund B, Kramer MS. Differences in late fetal death rates in association with determinants of small for gestational age fetuses: Population based cohort study. BMJ. 1998; 316(7143): 1483-7.

6. NICE. Intrauterine laser ablation of placental vessels for the treatment of twin-to-twin transfusion syndrome-information for the public. National Institute for Health and Clinical Excellence (NICE). 2006.

7. Day MC, Barton JR, O’Brien JM, Istwan NB, Sibai BM. The effect of fetal number on the development of hypertensive conditions of pregnancy. Obst Gynecol. 2005; 106: 927-31.

8. Malik MS, Rashid U. Complications of Twin Gestation. Biomedica. 1998; 14: 22-6.

9. Kahn B, Lumey LH, Zybert PA, Lorenz JM, ClearyGoldman J, D'Alton ME, et al. Prospective risk of fetal death in singleton, twin and triplet gestations: implications for practice. Obstet Gynecol. 2003; 102: 
Original Article | Journal of Gandaki Medical College-Nepal

685-92.

10. Ananth CV, Smulian JC, Demissie K, Vintzileos AM, Kunppel RA. Placental abruption among singleton and twin births in the United States: Risk factors profiles. Am J Epidemiol. 2001; 153: 771-8.

11. De Sutter P, Van der Elst J, Cetsier T, Dhont M. Single embryo transfer and multiple pregnancy rate reduction in IVF/ICIS: A 5 year appraisal. Reproductive Bio Medicine. Online 2003; 6(4): 464-9. 